\title{
FITOSSOCIOLOGIA DE UMA ÁREA DE PRESERVAÇÃO PERMANENTE NO LESTE DA AMAZÔNIA: INDICAÇẪO DE ESPÉCIES PARA RECUPERAÇÃO DE ÁREAS ALTERADAS
}

\author{
Klewton Adriano Oliveira Pinheiro*, João Olegário Pereira de Carvalho**, Beatriz Quanz***, \\ Luciana Maria de Barros Francez***, Gustavo Schwartz**** \\ *Eng. Florestal, M.Sc., Embrapa Amazônia Oriental - klewton.pinheiro@gmail.com \\ *** Eng. Florestal, Ph.D., Embrapa Amazônia Oriental - olegario.carvalho@gmail.com \\ ${ }^{* * *}$ Eng $^{\mathrm{a}}$. Florestal, M.Sc. - biaquanz@yahoo.com.br - lucianafrancez@yahoo.com.br \\ ${ }^{* * * *}$ Biólogo, M.Sc., Embrapa Amazônia Oriental - gustavo@cpatu.embrapa.br \\ Recebido para publicação: 16/05/2006 - Aceito para publicação: 25/01/2007
}

\begin{abstract}
Resumo
Neste artigo é apresentada a fitossociologia de um fragmento de Floresta Ombrófila Densa de 84 ha no nordeste do estado do Pará, com árvores de DAP $\geq 20 \mathrm{~cm}$. As espécies foram classificadas em grupos ecológicos em função das exigências de luz e quanto ao uso comercial. Os dados foram coletados em 12 parcelas de 1 ha aleatorizadas e subdivididas em 100 subparcelas de $10 \times 10 \mathrm{~m}$. Foram registrados 2.326 indivíduos distribuídos em 158 espécies, 107 gêneros e 43 famílias. As famílias Fabaceae e Lecythidaceae tiveram a maior riqueza e densidade na composição florística da área. A primeira apresentou o maior número de espécies (41), a segunda, o maior número de indivíduos (609), e ambas apresentaram alta dominância e freqüência. Quanto aos grupos ecológicos, as espécies tolerantes foram maioria $(63,29 \%$ ); quando considerada a classificação quanto ao uso econômico, as espécies comerciais predominaram $(41,15$ \%). Sugere-se que espécies com alta importância ecológica e interesse comercial sejam utilizadas para a recuperação de áreas alteradas. Entres as espécies encontradas, destacaram-se Pouteria heterosepala, Eschweilera amazonica, Eschweilera blanchetiana, Protium apiculatum, Guatteria poeppigiana, Sterculia pilosa, Inga brevialata e Licania heteromorpha. Entretanto, antes de qualquer ação empreendedora, devemse obter informações seguras sobre a ecologia e a silvicultura dessas espécies.

Palavras-chave: Composição florística; estrutura de floresta; grupos ecológicos; riqueza de espécies; mata ciliar.
\end{abstract}

\begin{abstract}
Phytosociology of a permanent preservation area in east of Amazon: indication of species for recovering altered areas. This paper presents the phytosociology of a fragment of a dense ombrophilic forest with 84 ha in Northeastern Pará state in trees with DBH $\geq 20 \mathrm{~cm}$. The species were classified in ecological groups in function of light demand and commercial use. The sampling was composed by 12 random 1 ha plots and divided in 100 subplots of $10 \mathrm{~m} \times 10 \mathrm{~m}$. It was recorded 2,326 individuals in 158 species, 107 genera and, 43 families. The families Fabaceae and Lecythidaceae had the highest richness and density in the floristic composition of the area. The first family presents the highest number of species (41) and the second, the highest number of individuals (609), and both of them present high dominance and frequency. As regards to the ecological groups, the species shade tolerant were majority (63.29\%) while in the economic use, the commercial species predominated (41.15\%). It suggests that species with high ecological importance and commercial interest be used for altered area recovering. Around sampled species, some of them are most important such as Pouteria heterosepala, Eschweilera amazonica, Eschweilera blanchetiana, Protium apiculatum, Guatteria poeppigiana, Sterculia pilosa, Inga brevialata and Licania heteromorpha. However, before any kind of investment it must get safe informations about ecology and silviculture of these species.

Keywords: Floristic composition; forest structure; ecological groups; species richness; riparian forest.
\end{abstract}

\section{INTRODUÇÃO}

A planície amazônica é coberta por um maciço florestal composto por várias formações vegetais em diferentes fisionomias intercaladas por rios, igarapés e lagos. Nesses ecossistemas, os processos ecológicos são complexos e dinâmicos, sendo que quaisquer intervenções para fins econômicos necessitam de prévio conhecimento sobre sua funcionalidade, a fim de alcançar-se um manejo sustentável 
(BENTES-GAMA, 2000; SANDEL; CARVALHO, 2000). O conhecimento dos processos ecológicos torna-se imperativo para o sucesso de um manejo sustentável em florestas tropicais (KAO; IIDA, 2006). Entre as informações ecológicas relevantes para um manejo adequado, estão os padrões ecológicos em que se distribuem as espécies florestais (SWAINE; WHITMORE, 1988), especialmente com relação às exigências de luz (DENSLOW; HARTSHORN, 1994; MYERS et al., 2000).

O desconhecimento de padrões ecológicos aliado a sistemáticas intervenções em florestas tropicais, especialmente na Amazônia brasileira, tem gerado grandes impactos ambientais. As suas conseqüências, em algumas regiões, requerem ações conservacionistas imediatas. Frente a essa problemática, torna-se necessário assegurar a conservação de remanescentes representativos dos diferentes ecossistemas (SANTANA et al., 2000). Os problemas ecológicos originados a partir dessas formas irracionais de exploração têm merecido crescentes discussões sobre a necessidade de planejamento e ordenamento das reservas vitais de água, solo, fauna e flora (MAGALHÃES; FERREIRA, 2000). Um dos grandes desafios para a sustentabilidade ambiental é a concentração de esforços e recursos para a conservação e recuperação de áreas naturais, consideradas estratégicas. Dentre elas, destacam-se as Áreas de Preservação Permanente (APP's), com papel vital para a manutenção das microbacias e imprescindíveis para a existência dos ecossistemas ali existentes (MAGALHÃES; FERREIRA, 2000). No Brasil, as APP's foram criadas pelo Código Florestal Brasileiro - CFB (Lei no 4771/1965), que determina que os direitos de propriedade são exercidos, porém com limitações (MEDAUAR, 2002). Uma APP consiste em uma faixa de preservação estabelecida em razão do relevo, geralmente localizada ao longo de cursos d'água, nascentes, topos e encostas de morros. Uma APP destina-se à manutenção da qualidade do solo, das águas e como corredor de biodiversidade (MILARÉ, 2000). No CFB, outros artigos reafirmam a necessidade de proteção para florestas em nascentes de rios, estabelecendo a obrigatoriedade de reflorestamento com espécies nativas em áreas alteradas. Somente com um conhecimento mais detalhado da composição florística natural de determinada área (RODRIGUES; NAVE, 2001), bem como das características ecológicas das espécies será possível o sucesso em ações mitigadoras de danos ambientais.

O objetivo deste estudo foi conhecer a composição florística e a estrutura horizontal de um fragmento de floresta ombrófila densa em área de preservação permanente no leste da Amazônia. Para cada espécie encontrada, definiu-se o modo de resposta de crescimento com relação à luz (grupo ecológico) e a classificação quanto ao interesse comercial. Com esses resultados, espera-se contribuir com informações para planos de manejo de florestas com composição florística e estrutura semelhantes, assim como na recuperação de áreas que, mesmo sendo de preservação permanente, sofreram danos antrópicos.

\section{MATERIAL E MÉTODOS}

\section{Caracterização da área de estudo}

$\mathrm{O}$ estudo foi desenvolvido em uma área de Floresta Ombrófila Densa na fazenda Rio Capim $\left(03^{\circ} 30^{\prime}-03^{\circ} 45^{\prime} \mathrm{S}\right.$ e $\left.48^{\circ} 30^{\prime}-48^{\circ} 45^{\prime} \mathrm{W}\right)$, pertencente à empresa florestal Cikel Brasil Verde S/A, no município de Paragominas, nordeste do estado do Pará (Figura 1), na microrregião homogênea Guajarina (WATRIN; ROCHA, 1991).

Na região, segundo a classificação de Köeppen, o clima é do tipo Am, com precipitação pluviométrica em torno de $2.600 \mathrm{~mm}$ anuais. O período chuvoso ocorre entre novembro e abril, com temperaturas médias anuais entre 24 e $26{ }^{\circ} \mathrm{C}$, e a umidade relativa do ar em torno de 80 a $85 \%$ (SUDAM, 1993). O terreno mostra uma topografia variável, de plana a suavemente ondulada, com locais bastante acidentados. Os principais solos são Latossolos Amarelos, Argissolos Amarelos e Gleissolos (SILVA, 1997), com baixa fertilidade e boas propriedades físicas, como profundidade e drenagem (MORAIS-CRUIA et al., 1999). Segundo Veloso et al. (1991), na área compreendida pela fazenda Rio Capim existem dois ambientes fitoecológicos (Floresta Ombrófila Densa e Floresta Ombrófila Densa Aluvial), com mananciais de água pertencentes às bacias dos rios Capim, Surubijú e Gurupi (WATRIN; ROCHA, 1991).

\section{Amostragem}

A área onde foi realizada a amostragem (84 ha) corresponde à Unidade de Trabalho (UT) 16 da Unidade de Produção Anual (UPA) 06 do Plano de Manejo Florestal da fazenda Rio Capim. Nela foi realizado um inventário de prospecção $(100 \%)$ com o objetivo de conduzir a exploração madeireira. Após o 
microzoneamento, conforme o CFB, constatou-se que mais de $80 \%$ da UT era de preservação permanente, devido à existência de pequenos cursos d'água, grotas secas e terrenos com declividade acentuada.

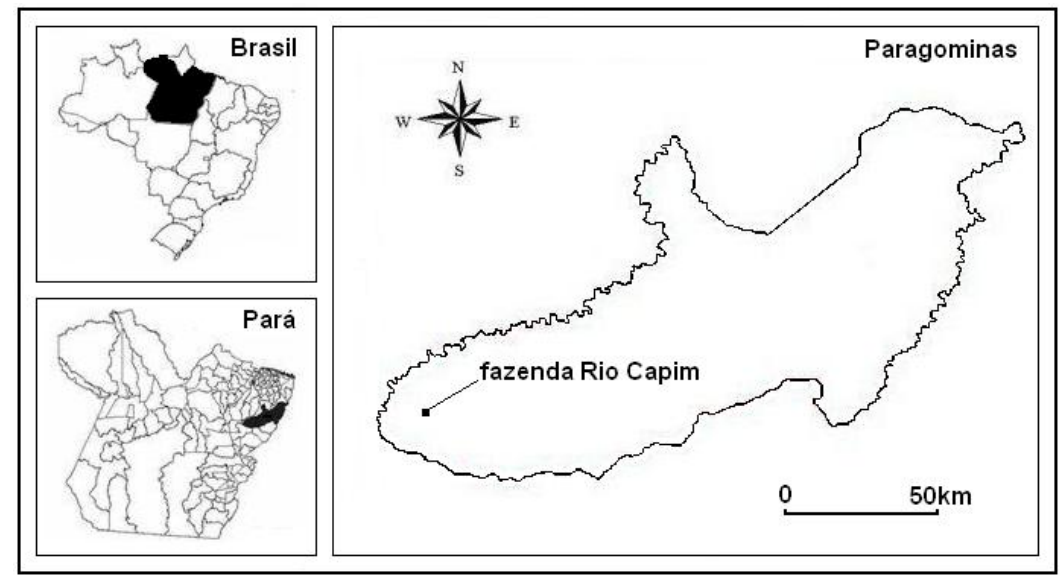

Figura 1. Localização da fazenda Rio Capim no oeste do município de Paragominas, estado do Pará, Brasil.

Figure 1. Location of Rio Capim farm in Western Paragominas municipality, Pará state, Brazil.

Os dados foram coletados segundo o método de Silva e Lopes (1984) e Silva et al. (2005), com algumas adaptações. Foram estabelecidas ao acaso 12 parcelas permanentes de 1 ha, divididas em 100 subparcelas de $10 \times 10 \mathrm{~m}$, totalizando 1.200 subparcelas, marcadas com piquetes de $1,5 \mathrm{~m}$ de altura. Nessas subparcelas foram medidas as árvores com DAP (diâmetro a $1,30 \mathrm{~m}$ do solo) $\geq 20 \mathrm{~cm}$ de todas as espécies, exceto as das famílias Arecaceae e Cecropiaceae. Essas famílias não apresentam interesse comercial pelo setor madeireiro da região e, em geral, não são incluídas em inventários de prospecção. Cada árvore foi medida com fita diamétrica e numerada com uma plaqueta de alumínio, fixada com prego a uma altura de $1,5 \mathrm{~m}$ do solo e marcada com tinta vermelha.

Os indivíduos mais comuns foram identificados no campo com o auxílio de um parataxônomo. Quando a identificação em campo foi impossível, amostras de material fértil ou vegetativo foram levadas para a identificação no Herbário IAN, no Laboratório de Botânica da Embrapa Amazônia Oriental. Para a identificação das espécies, adotou-se o sistema de classificação do Angiosperm Phylogeny Group (APG) atualizado em APG II (2003).

\section{Análises}

Para as análises dos dados, foram considerados, conforme Souza (1973), sete parâmetros. Densidade absoluta (DA), como sendo a relação do total de indivíduos de uma determinada espécie por unidade de área. Densidade relativa (DR), representando a participação de uma determinada espécie em relação à abundância total (todas as árvores), em porcentagem. Freqüência (Fr), como sendo a distribuição de cada espécie, em termos percentuais, na área. Freqüência absoluta (FA), obtida da percentagem de subparcelas em que ocorreu uma determinada espécie. Freqüência relativa (FR), obtida da percentagem de freqüência absoluta das espécies na área em relação ao total de freqüência absoluta (todas as espécies). Dominância absoluta (DoA), medida da projeção total do corpo da planta no solo, determinada através da área basal, ou seja, a soma das áreas transversais das plantas de uma determinada espécie (FÖRSTER, 1973). Dominância relativa (DoR), considerada como sendo a percentagem de área basal de cada espécie em relação à área basal total. Segundo Souza (1973), o valor de importância é determinado pela combinação em uma expressão de densidade com dominância e freqüência, em termos relativos, representado na equação a seguir:

$\mathrm{VI}=\mathrm{DR}+\mathrm{DoR}+\mathrm{FR}$

Sendo: $\mathrm{VI}=$ valor de importância; $\mathrm{DR}=$ densidade relativa; $\mathrm{DoR}=$ dominância relativa; $\mathrm{FR}=$ freqüência relativa. 
Para a análise dos dados e o cálculo dos parâmetros fitossociológicos, foram utilizados os programas SFC e FITOPAC 1 (SHEPHERD, 1994; YARED et al., 2000).

\section{Grupos ecológicos}

As espécies foram classificadas em grupos ecológicos, de acordo com Whitmore (1989a, 1989b) e Carvalho (1992), em espécies tolerantes à sombra e espécies demandantes de luz (ou intolerantes à sombra). A classificação das espécies em usos comerciais foi feita com base em características tecnológicas da madeira e seu mercado (nacional e internacional), de acordo com Brasil (1988), BrasilSudam (1981), Silva e Rosa (1989) e Souza et al. (1997).

\section{RESULTADOS}

\section{Composição florística}

A amostragem incluiu 2.326 indivíduos com DAP $\geq 20 \mathrm{~cm}$, distribuídos em 158 espécies, 107 gêneros e 43 famílias. Das 158 espécies amostradas, cinco foram identificadas em nível de gênero e uma não pôde ser identificada, nem mesmo em nível de família. As doze famílias com maior riqueza de espécies foram Fabaceae, Moraceae, Sapotaceae, Lecythidaceae, Lauraceae, Annonaceae, Meliaceae, Burseraceae, Anacardiaceae, Apocynaceae, Rubiaceae e Euphorbiaceae, que, juntas, somaram 106 espécies ou 67,09 \% da riqueza específica da área amostrada. As dez famílias botânicas com maior número de indivíduos que, juntas, somaram 1.948 indivíduos (83,75 \% do total), foram: Lecythidaceae (26,18 \%); Sapotaceae $(20,42$ $\%$ ); Fabaceae (14,45 \%); Burseraceae (4,73\%); Chrysobalanaceae (4,43\%); Moraceae (3,74 \%); Lauraceae $(3,01 \%)$; Humiriaceae $(2,54 \%)$; Annonaceae $(2,36 \%)$ e Meliaceae $(1,89 \%)$. Ao levar em conta tanto o número de indivíduos quanto o de espécies, as duas famílias mais importantes foram Fabaceae e Lecythidaceae. A família Fabaceae foi representada por 24 gêneros e 41 espécies (Tabela 1).

Quatorze famílias (Araliaceae, Bixaceae, Combretaceae, Elaeocarpaceae, Malpighiaceae, Myristicaceae, Nyctaginaceae, Ochnaceae, Olacaceae, Opiliaceae, Quiinaceae, Sapindaceae, Tiliaceae e Vochysiaceae) foram representadas por apenas uma espécie $(8,86 \%$ do total de espécies e 32,56 \% do total de famílias). Desse conjunto, maior raridade foi apresentada pelas famílias Araliaceae, Ochnaceae, Opiliaceae, Quiinaceae e Vochysiaceae, as quais foram representadas por apenas um indivíduo.

Tabela 1. Composição florística (famílias e espécies). Grupo ecológico (GE): tolerante (To), intolerante (It) e indefinido (In). Grau de comercialização da madeira (GC): comercial (Co), potencial (Po), não-comercial (Nc) e indefinido (In). Densidade absoluta (DA), dominância absoluta (DoA), freqüência absoluta (FA) e valor de importância (VI) das espécies encontradas em uma amostra de 12 ha de floresta natural na fazenda Rio Capim, Paragominas (PA), considerando indivíduos com DAP $\geq 20 \mathrm{~cm}$ (árvores).

Table 1. Floristic composition (botanical families and species). Ecological group (GE): tolerant (To), no-tolerant (It) and unknown (In). Level of timber market (GC): commercial (Co), potential (Po), no-commercial (Nc) and unknown (In). Absolute density (DA), absolute dominance (DoA), absolute frequency (FA) and value of importance (VI) of species found in natural forest sample of 12 ha in Rio Capim farm, Paragominas (PA), considering individuals with $\mathrm{DBH} \geq 20 \mathrm{~cm}$ (trees).

\begin{tabular}{|c|c|c|c|c|c|c|}
\hline Família/Espécie & GE & GC & DA(ind./ha) & $\operatorname{Do} A\left(\mathbf{m}^{2}\right)$ & FA(\%) & VI \\
\hline \multicolumn{7}{|l|}{ Anacardiaceae } \\
\hline Anacardium giganteum $W$. Hancock ex Engl. & It & $\mathrm{Co}$ & 0,3 & 0,121 & 25,00 & 1,08 \\
\hline Astronium gracile Engl. & To & $\mathrm{Co}$ & 0,7 & 0,100 & 50,00 & 1,64 \\
\hline Spondias mombin L. & To & $\mathrm{Co}$ & 0,6 & 0,058 & 50,00 & 1,41 \\
\hline Tapirira guianensis Aubl. & It & Po & 0,8 & 0,088 & 58,33 & 1,77 \\
\hline \multicolumn{7}{|l|}{ Annonaceae } \\
\hline Duguetia echinophora R. E. Fr. & To & $\mathrm{Nc}$ & 0,7 & 0,029 & 58,33 & 1,47 \\
\hline Fusaea longifolia (Aubl.) Saff. & In & In & 0,2 & 0,015 & 16,67 & 0,44 \\
\hline Guatteria ovalifolia R. E. Fr. & To & $\mathrm{Nc}$ & 0,8 & 0,067 & 58,33 & 1,72 \\
\hline Guatteria poeppigiana Mart. & To & Po & 2,8 & 0,219 & 100 & 4,13 \\
\hline Xylopia benthami R. E. Fr. & In & In & 0,1 & 0,005 & 8,33 & 0,21 \\
\hline \multicolumn{7}{|l|}{ Apocynaceae } \\
\hline Aspidosperma desmanthum Benth. ex Müll. Arg. & To & $\mathrm{Co}$ & 0,1 & 0,048 & 8,33 & 0,40 \\
\hline Aspidosperma megalocarpon Müll. Arg. & To & Po & 0,3 & 0,029 & 25,00 & 0,69 \\
\hline Aspidosperma rigidum Rusby & To & $\mathrm{Nc}$ & 0,7 & 0,301 & 50,00 & 2,52 \\
\hline Himatanthus sucuuba (Spruce ex Müll. Arg.) Woodson & To & $\mathrm{Co}$ & 0,1 & 0,005 & 8,33 & 0,21 \\
\hline
\end{tabular}




\begin{tabular}{|c|c|c|c|c|c|c|}
\hline Família/Espécie & GE & GC & DA(ind./ha) & $\operatorname{DoA}\left(\mathbf{m}^{2}\right)$ & FA(\%) & VI \\
\hline \multicolumn{7}{|l|}{ Araliaceae } \\
\hline Schefflera morototoni (Aubl.) Maguire, Steyerm. \& Fr. & It & $\mathrm{Co}$ & 0,1 & 0,023 & 8,33 & 0,29 \\
\hline \multicolumn{7}{|l|}{ Bignoniaceae } \\
\hline Jacaranda copaia (Aubl.) D. Don & It & Co & 1,0 & 0,107 & 66,67 & 2,13 \\
\hline Tabebuia impetiginosa (Mart. ex DC.) Standl. & It & $\mathrm{Co}$ & 0,1 & 0,056 & 8,33 & 0,43 \\
\hline \multicolumn{7}{|l|}{ Bixaceae } \\
\hline Bixa arborea Huber & It & Po & 0,8 & 0,083 & 33,33 & 1,32 \\
\hline \multicolumn{7}{|l|}{ Bombacaceae } \\
\hline Bombax munguba Mart. \& Zucc. & It & $\mathrm{Co}$ & 0,2 & 0,013 & 16,67 & 0,43 \\
\hline Bombax paraensis Ducke & To & $\mathrm{Nc}$ & 0,1 & 0,004 & 8,33 & 0,20 \\
\hline \multicolumn{7}{|l|}{ Boraginaceae } \\
\hline Cordia alliodora (Ruiz \& Pav.) Cham. Oken & It & Co & 0,4 & 0,023 & 33,33 & 0,88 \\
\hline Cordia bicolor A. DC. & It & $\mathrm{Co}$ & 1,1 & 0,106 & 50,00 & 1,88 \\
\hline Cordia goeldiana Huber & It & $\mathrm{Co}$ & 0,4 & 0,043 & 25,00 & 0,83 \\
\hline \multicolumn{7}{|l|}{ Burseraceae } \\
\hline Protium apiculatum Swart & To & Po & 5,3 & 0,781 & 100 & 7,88 \\
\hline Protium opacum Swart & To & Po & 0,2 & 0,010 & 16,67 & 0,42 \\
\hline Protium subserratum (Engl.) Engl. & To & Po & 2,9 & 0,525 & 91,67 & 5,38 \\
\hline Trattinnickia rhoifolia Willd. & To & $\mathrm{Co}$ & 0,8 & 0,140 & 50,00 & 1,86 \\
\hline \multicolumn{7}{|l|}{ Caryocaraceae } \\
\hline Caryocar glabrum (Aubl.) Pers. & It & $\mathrm{Co}$ & 0,4 & 0,096 & 33,33 & 1,21 \\
\hline Caryocar villosum (Aubl.) Pers. & It & $\mathrm{Co}$ & 0,1 & 0,034 & 8,33 & 0,33 \\
\hline \multicolumn{7}{|l|}{ Celastraceae } \\
\hline Goupia glabra Aubl. & It & Co & 1,4 & 0,416 & 75,00 & 3,84 \\
\hline Maytenus pruinosa Reissek & To & $\mathrm{Nc}$ & 0,2 & 0,022 & 16,67 & 0,47 \\
\hline \multicolumn{7}{|l|}{ Chrysobalanaceae } \\
\hline Couepia bracteosa Benth. & To & $\mathrm{Nc}$ & 0,6 & 0,040 & 33,33 & 1,05 \\
\hline Licania heteromorpha Benth. & To & $\mathrm{Nc}$ & 2,8 & 0,257 & 83,33 & 4,02 \\
\hline Licania incana Aubl. & To & $\mathrm{Nc}$ & 5,2 & 0,566 & 75,00 & 6,43 \\
\hline \multicolumn{7}{|l|}{ Combretaceae } \\
\hline \multirow{2}{*}{\multicolumn{7}{|c|}{ Clusiaceae }} \\
\hline & & & & & & \\
\hline Symphonia globulifera L.f. & It & $\mathrm{Co}$ & 1,3 & 0,161 & 58,33 & 2,35 \\
\hline Vismia guianensis (Aubl.) Pers. & It & $\mathrm{Nc}$ & 0,2 & 0,009 & 16,67 & 0,41 \\
\hline \multicolumn{7}{|l|}{ Ebenaceae } \\
\hline Diospyros praetermissa Sandwith & To & $\mathrm{Nc}$ & 0,1 & 0,003 & 8,33 & 0,20 \\
\hline Diospyros sp. & In & In & 0,4 & 0,021 & 25,00 & 0,74 \\
\hline Elaeocarpaceae & & & & & & \\
\hline Sloanea froesii Earle Sm. & It & $\mathrm{Nc}$ & 1,5 & 0,129 & 66,67 & 2,48 \\
\hline Euphorbiaceae & & & & & & \\
\hline Glycydendron amazonicum Ducke & To & Po & 0,1 & 0,013 & 8,33 & 0,24 \\
\hline Hevea brasiliensis (Willd. ex A. Juss.) Müll. Agr. & It & Po & 0,3 & 0,039 & 25,00 & 0,77 \\
\hline Maprounea guianensis Aubl. & To & $\mathrm{Nc}$ & 0,2 & 0,010 & 16,67 & 0,41 \\
\hline Sapium marmieri Huber & To & Po & 0,1 & 0,003 & 8,33 & 0,20 \\
\hline Fabaceae & & & & & & \\
\hline Abarema jupunba (Willd.) Britton \& Killip & It & Co & 1,6 & 0,193 & 58,33 & 2,66 \\
\hline Bowdichia nitida Spruce ex Benth. & To & $\mathrm{Co}$ & 0,1 & 0,028 & 8,33 & 0,31 \\
\hline Dialium guianense (Aubl.) Sandwith & To & $\mathrm{Co}$ & 0,1 & 0,013 & 8,33 & 0,24 \\
\hline Diplotropis purpurea (Rich.) Amshoff & To & $\mathrm{Co}$ & 0,2 & 0,068 & 16,67 & 0,67 \\
\hline Diplotropis sp. & In & In & 0,5 & 0,028 & 50,00 & 1,24 \\
\hline Dipteryx odorata (Aubl.) Willd. & It & $\mathrm{Co}$ & 0,1 & 0,012 & 8,33 & 0,24 \\
\hline Enterolobium contortisiliquum (Vell.) Morong & It & Co & 0,2 & 0,011 & 16,67 & 0,42 \\
\hline Enterolobium maximum Ducke & It & Co & 0,3 & 0,029 & 16,67 & 0,59 \\
\hline Enterolobium schomburgkii (Benth.) Benth & It & Co & 0,3 & 0,071 & 25,00 & 0,87 \\
\hline Eperua bijuga Mart. ex Benth. & To & $\mathrm{Nc}$ & 0,8 & 0,034 & 50,00 & 1,44 \\
\hline Eperua schomburgkiana Benth. & To & Co & 0,3 & 0,061 & 33,33 & 1,01 \\
\hline Hymenaea courbaril L. & To & $\mathrm{Co}$ & 0,6 & 0,145 & 50,00 & 1,79 \\
\hline Hymenaea parvifolia Huber & To & $\mathrm{Co}$ & 0,3 & 0,041 & 25,00 & 0,78 \\
\hline Inga alba (Sw.) Willd. & To & $\mathrm{Nc}$ & 0,1 & 0,005 & 8,33 & 0,21 \\
\hline Inga paraensis Ducke & To & Co & 3,5 & 0,384 & 91,67 & 5,06 \\
\hline Inga brevialata Ducke & It & $\mathrm{Nc}$ & 6,0 & 0,412 & 100 & 6,61 \\
\hline Macrolobium latifolium Vogel & To & Co & 3,8 & 0,297 & 75,00 & 4,56 \\
\hline Ormosia flava (Ducke) Rudd & It & Po & 0,5 & 0,030 & 41,67 & 1,10 \\
\hline Ormosia sp. & In & In & 0,2 & 0,036 & 16,67 & 0,54 \\
\hline Parkia nitida Miq. & It & Co & 0,1 & 0,016 & 8,33 & 0,26 \\
\hline Parkia gigantocarpa Ducke & It & $\mathrm{Co}$ & 0,1 & 0,016 & 8,33 & 0,26 \\
\hline
\end{tabular}

FLORESTA, Curitiba, PR, v. 37, n. 2, mai./ago. 2007. 


\begin{tabular}{|c|c|c|c|c|c|c|}
\hline Família/Espécie & GE & GC & DA(ind./ha) & $\operatorname{DoA}\left(\mathbf{m}^{2}\right)$ & FA(\%) & VI \\
\hline Parkia multijuga Benth. & It & Co & 0,7 & 0,161 & 50,00 & 1,91 \\
\hline Parkia pendula (Willd.) Benth. ex Walp. & It & Co & 0,8 & 0,044 & 50,00 & 1,48 \\
\hline Peltogyne lecointei Ducke & To & $\mathrm{Co}$ & 0,3 & 0,130 & 25,00 & 1,13 \\
\hline Piptadenia suaveolens Miq. & It & Po & 1,8 & 0,488 & 75,00 & 4,33 \\
\hline Pithecellobium racemosum Ducke & It & Co & 0,8 & 0,064 & 75,00 & 1,99 \\
\hline Pithecellobium scandens Ducke & It & Po & 0,2 & 0,008 & 16,67 & 0,41 \\
\hline Poecilanthe effusa (Huber) Ducke & To & $\mathrm{Nc}$ & 0,1 & 0,003 & 8,33 & 0,20 \\
\hline Pterocarpus amazonicus Huber & To & Po & 0,1 & 0,008 & 8,33 & 0,22 \\
\hline Schizolobium amazonicum Huber ex Ducke & It & Co & 0,1 & 0,008 & 8,33 & 0,22 \\
\hline Sclerolobium guianense Benth. & It & Po & 0,3 & 0,033 & 25,00 & 0,70 \\
\hline Sclerolobium paraense Huber & It & Po & 0,7 & 0,204 & 50,00 & 2,09 \\
\hline Stryphnodendron pulcherrimum (Willd.) Hochr. & It & Po & 0,3 & 0,027 & 25,00 & 0,67 \\
\hline Stryphnodendron angustum Benth. & To & $\mathrm{Nc}$ & 0,2 & 0,009 & 16,67 & 0,41 \\
\hline Swartzia aptera DC. & To & $\mathrm{Nc}$ & 0,1 & 0,026 & 8,33 & 0,30 \\
\hline Swartzia stipulifera Harms & To & Po & 0,3 & 0,048 & 33,33 & 0,96 \\
\hline Tachigali alba Ducke & It & Co & 0,1 & 0,033 & 8,33 & 0,33 \\
\hline Tachigali myrmecophila (Ducke) Ducke & It & $\mathrm{Co}$ & 1,0 & 0,209 & 66,67 & 2,57 \\
\hline Tachigali paniculata Aubl. & To & $\mathrm{Co}$ & 0,1 & 0,022 & 8,33 & 0,28 \\
\hline Vataireopsis speciosa Ducke & It & Po & 0,2 & 0,006 & 16,67 & 0,40 \\
\hline Zollernia paraensis Huber & In & In & 0,7 & 0,074 & 41,67 & 1,38 \\
\hline \multicolumn{7}{|l|}{ Flacourtiaceae } \\
\hline Casearia javitensis Kunth & To & $\mathrm{Nc}$ & 0,1 & 0,005 & 8,33 & 0,21 \\
\hline Laetia procera (Poepp.) Eichler & It & Co & 1,1 & 0,193 & 66,67 & 2,55 \\
\hline \multicolumn{7}{|l|}{ Humiriaceae } \\
\hline Endopleura uchi (Huber) Cuatrec. & To & Co & 0,2 & 0,012 & 16,67 & 0,42 \\
\hline Sacoglottis guianensis Benth. & To & $\mathrm{Co}$ & 0,5 & 0,043 & 25,00 & 0,87 \\
\hline Sacoglottis amazonica Mart. & To & $\mathrm{Nc}$ & 4,3 & 0,539 & 100 & 6,27 \\
\hline \multicolumn{7}{|l|}{ Lauraceae } \\
\hline Aniba canelilla (Kunth) Mez & To & Co & 0,2 & 0,049 & 16,67 & 0,62 \\
\hline Aniba guianensis Aubl. & To & Co & 0,3 & 0,070 & 25,00 & 0,91 \\
\hline Licaria cannella (Meisn.) Kosterm. & To & $\mathrm{Co}$ & 2,7 & 0,316 & 91,67 & 4,33 \\
\hline Mezilaurus itauba (Meisn.) Taub. ex Mez & To & $\mathrm{Co}$ & 0,1 & 0,025 & 8,33 & 0,29 \\
\hline Ocotea sp. & In & In & 2,1 & 0,294 & 91,67 & 4.37 \\
\hline Ocotea rubra $\mathrm{Mez}$ & To & Po & 0,5 & 0,093 & 41,67 & 1,38 \\
\hline \multicolumn{7}{|l|}{ Lecythidaceae } \\
\hline Couratari oblongifolia Ducke \& R. Knuth. & To & Po & 0,3 & 0,108 & 25,00 & 1,03 \\
\hline Eschweilera amara (Aubl.) Nied. & To & $\mathrm{Nc}$ & 1,3 & 0,091 & 66,67 & 2,19 \\
\hline Eschweilera amazonica $\mathrm{R}$. Knuth & To & $\mathrm{Nc}$ & 21,4 & 1,235 & 100 & 18,17 \\
\hline Eschweilera blanchetiana (O. Berg) Miers & To & $\mathrm{Nc}$ & 26,3 & 2,456 & 100 & 26,01 \\
\hline Eschweilera odora (Poepp. ex O. Berg) Miers & To & $\mathrm{Nc}$ & 0,2 & 0,010 & 8,33 & 0,27 \\
\hline Eschweilera coriacea (DC.) S. A. Mori & To & $\mathrm{Nc}$ & 0,7 & 0,149 & 58,33 & 1,47 \\
\hline Lecythis lurida (Miers.) S. A. Mori & To & Co & 0,4 & 0,119 & 33,33 & 1,31 \\
\hline Lecythis usitata Miers & To & Co & 0,3 & 0,194 & 25,00 & 1,45 \\
\hline \multicolumn{7}{|l|}{ Malpighiaceae } \\
\hline Byrsonima crispa A. Juss. & It & $\mathrm{Nc}$ & 0,3 & 0,018 & 25,00 & 0,64 \\
\hline \multicolumn{7}{|l|}{ Melastomataceae } \\
\hline Bellucia dichotoma Cogn. & It & $\mathrm{Nc}$ & 0,1 & 0,003 & 8,33 & 0,20 \\
\hline Mouriria plasschaerti Pulle & To & Po & 0,3 & 0,014 & 25,00 & 0,64 \\
\hline \multicolumn{7}{|l|}{ Meliaceae } \\
\hline Carapa guianensis Aubl. & To & Co & 2,7 & 0,305 & 75,00 & 4,00 \\
\hline Guarea kunthiana A. Juss. & To & Po & 0,3 & 0,024 & 25,00 & 0,66 \\
\hline Guarea guidonia (L.) Sleumer & To & $\mathrm{Nc}$ & 0,3 & 0,060 & 16,67 & 0,68 \\
\hline Trichilia paraensis C.DC. & To & $\mathrm{Nc}$ & 0,4 & 0,020 & 33,33 & 0,87 \\
\hline \multicolumn{7}{|l|}{ Moraceae } \\
\hline Bagassa guianensis Aubl. & It & Co & 0,1 & 0,048 & 8,33 & 0,40 \\
\hline Brosimum guianense (Aubl.) Huber. & To & $\mathrm{Co}$ & 0,5 & 0,096 & 41,67 & 1,39 \\
\hline Brosimum lactescens (S. Moore) C. C. Berg. & To & Po & 0,2 & 0,026 & 16,67 & 0,49 \\
\hline Brosimum lanciferum Ducke & To & $\mathrm{Nc}$ & 0,2 & 0,010 & 8,33 & 0,27 \\
\hline Brosimum ovatifolium Ducke & To & Po & 0,5 & 0,109 & 41,67 & 1,45 \\
\hline Brosimum paraense Huber & To & $\mathrm{Nc}$ & 1,0 & 0,070 & 16,67 & 1,11 \\
\hline Brosimum parinarioides Ducke & To & Co & 0,3 & 0,034 & 25,00 & 0,75 \\
\hline Helicostylis pedunculata Benoist & To & Po & 0,2 & 0,012 & 16,67 & 0,42 \\
\hline Maquira guianensis Aubl. & To & Po & 0,2 & 0,019 & 16,67 & 0,45 \\
\hline Perebea guianensis Aubl. & To & Po & 0,4 & 0,030 & 41,67 & 1,06 \\
\hline Perebea mollis (Poepp. \& Endl.) Huber & To & $\mathrm{Nc}$ & 0,2 & 0,012 & 16,67 & 0,42 \\
\hline Sahagunia racemifera Huber & To & $\mathrm{Nc}$ & 1,4 & 0,098 & 83,33 & 2,59 \\
\hline
\end{tabular}




\begin{tabular}{|c|c|c|c|c|c|c|}
\hline Família/Espécie & GE & GC & DA(ind./ha) & $\operatorname{DoA}\left(\mathrm{m}^{2}\right)$ & FA(\%) & VI \\
\hline \multicolumn{7}{|l|}{ Myristicaceae } \\
\hline Virola melinoni (Benoist) A. C. Sm. & To & $\mathrm{Co}$ & 0,8 & 0,114 & 58,33 & 1,93 \\
\hline \multicolumn{7}{|l|}{ Myrtaceae } \\
\hline Eugenia lambertiana DC. & To & $\mathrm{Nc}$ & 0,1 & 0,003 & 8,33 & 0,20 \\
\hline Eugenia paraensis O. Berg. & To & $\mathrm{Nc}$ & 0,1 & 0,004 & 8,33 & 0,20 \\
\hline Myrcia sp. & In & In & 0,3 & 0,013 & 16,67 & 0,47 \\
\hline \multicolumn{7}{|l|}{ Nyctaginaceae } \\
\hline Neea floribunda Poepp. \& Endl. & To & $\mathrm{Nc}$ & 3,4 & 0,272 & 83,33 & 4,38 \\
\hline \multicolumn{7}{|l|}{ Ochnaceae } \\
\hline Gomphia aquatica Kunth & To & $\mathrm{Nc}$ & 0,1 & 0,005 & 8,33 & 0,21 \\
\hline \multicolumn{7}{|l|}{ Olacaceae } \\
\hline Minquartia guianensis Aubl. & To & $\mathrm{Co}$ & 0,2 & 0,007 & 16,67 & 0,40 \\
\hline \multicolumn{7}{|l|}{ Opiliaceae } \\
\hline Agonandra brasiliensis Miers ex Benth. \& Hook. f. & In & In & 0,1 & 0,006 & 8,33 & 0,21 \\
\hline \multicolumn{7}{|l|}{ Quiinaceae } \\
\hline Lacunaria jenmanii (Oliv.) Ducke & To & $\mathrm{Nc}$ & 0,1 & 0,004 & 8,33 & 0,20 \\
\hline \multicolumn{7}{|l|}{ Rubiaceae } \\
\hline Capirona huberiana Ducke & To & $\mathrm{Nc}$ & 0,1 & 0,004 & 8,33 & 0,20 \\
\hline Chimarrhis turbinata DC. & To & $\mathrm{Nc}$ & 0,3 & 0,020 & 25,00 & 0,69 \\
\hline Coussarea paniculata (Vahl.) Standl. & To & $\mathrm{Nc}$ & 0,1 & 0,004 & 8,33 & 0,20 \\
\hline Duroia sprucei Rusby & To & $\mathrm{Nc}$ & 0,1 & 0,003 & 8,33 & 0,20 \\
\hline \multicolumn{7}{|l|}{ Rutaceae } \\
\hline Euxylophora paraensis Huber & It & $\mathrm{Co}$ & 0,7 & 0,309 & 41,67 & 2,41 \\
\hline Zanthoxylum hermaphroditum Willd. & It & $\mathrm{Nc}$ & 0,1 & 0,003 & 8,33 & 0,20 \\
\hline \multicolumn{7}{|l|}{ Sapindaceae } \\
\hline Talisia longifolia (Benth.) Radlk. & To & $\mathrm{Nc}$ & 0,3 & 0,026 & 25,00 & 0,71 \\
\hline \multicolumn{7}{|l|}{ Sapotaceae } \\
\hline Chrysophyllum oppositum (Ducke) Ducke & To & Po & 0,4 & 0,057 & 16,67 & 0,75 \\
\hline Manilkara huberi (Ducke) Chevalier & To & $\mathrm{Co}$ & 1,2 & 0,338 & 58,33 & 3,08 \\
\hline Manilkara paraensis (Huber) Standl. & To & $\mathrm{Co}$ & 4,6 & 1,008 & 91,67 & 8,35 \\
\hline Micropholis venulosa (Mart. \& Eichler) Pierre & To & $\mathrm{Co}$ & 6,6 & 0,668 & 100 & 8,03 \\
\hline Pouteria bilocularis (H. Winkl.) Baehni & To & $\mathrm{Co}$ & 0,9 & 0,096 & 33,33 & 1,46 \\
\hline Pouteria egregia Sandwith & To & Po & 0,3 & 0,041 & 25,00 & 0,74 \\
\hline Pouteria guianensis Aubl. & To & $\mathrm{Co}$ & 0,3 & 0,056 & 16,67 & 0,66 \\
\hline Pouteria heterosepala Pires & To & $\mathrm{Co}$ & 22,3 & 2,799 & 100 & 25,40 \\
\hline Pouteria macrophylla (Lam.) Eyma & To & Po & 0,6 & 0,030 & 41,67 & 1,15 \\
\hline Syzygiopsis oppositifolia Ducke & To & $\mathrm{Nc}$ & 2,6 & 0,311 & 83,33 & 4,12 \\
\hline \multicolumn{7}{|l|}{ Simaroubaceae } \\
\hline Simaba cedron Planch. & To & $\mathrm{Nc}$ & 0,4 & 0,020 & 33,33 & 0,87 \\
\hline Simarouba amara Aubl. & It & $\mathrm{Co}$ & 0,1 & 0,029 & 8,33 & 0,31 \\
\hline \multicolumn{7}{|l|}{ Sterculiaceae } \\
\hline Sterculia pilosa Ducke & It & Po & 2,6 & 0,248 & 91,67 & 3,99 \\
\hline Theobroma speciosum Willd. ex. Spreng. & To & $\mathrm{Nc}$ & 0,1 & 0,004 & 8,33 & 0,20 \\
\hline \multicolumn{7}{|l|}{ Tiliaceae $T$ - } \\
\hline Apeiba albiflora Ducke & It & Po & 2,1 & 0,297 & 91,67 & 3,95 \\
\hline \multicolumn{7}{|l|}{ Violaceae } \\
\hline Rinorea flavescens (Aubl.) Kuntze & To & $\mathrm{Nc}$ & 0,1 & 0,003 & 8,33 & 0,20 \\
\hline Rinorea guianensis Aubl. & To & $\mathrm{Nc}$ & 3,2 & 0,181 & 66,67 & 3,57 \\
\hline Rinorea macrocarpa (C. Mart. ex Eichler) Kuntze & To & $\mathrm{Nc}$ & 0,2 & 0,006 & 16,67 & 0,40 \\
\hline \multicolumn{7}{|l|}{ Vochysiaceae } \\
\hline Qualea albiflora Warm. & To & $\mathrm{Co}$ & 0,1 & 0,069 & 8,33 & 0,53 \\
\hline \multicolumn{7}{|l|}{ Família não identificada } \\
\hline Não Identificada & In & In & 0,2 & 0,012 & 16,67 & 0,43 \\
\hline
\end{tabular}

\section{Estrutura}

As dez espécies que apresentaram os maiores valores de dominância somaram uma área basal de $10,99 \mathrm{~m}^{2} \cdot \mathrm{ha}^{-1}$. Pouteria heterosepala foi a que apresentou maior dominância absoluta $\left(2,80 \mathrm{~m}^{2} \cdot \mathrm{ha}^{-1} ; 267\right.$ indivíduos), seguida por Eschweilera blanchetiana $\left(2,46 \mathrm{~m}^{2} . \mathrm{ha}^{-1} ; 315\right.$ indivíduos); Eschweilera amazonica $\left(1,23 \mathrm{~m}^{2} . \mathrm{ha}^{-1} ; 257\right.$ indivíduos); Manilkara paraensis $\left(1,01 \mathrm{~m}^{2} . \mathrm{ha}^{-1} ; 55\right.$ indivíduos); Protium apiculatum $(0,78$ $\mathrm{m}^{2} . \mathrm{ha}^{-1} ; 64$ indivíduos); Micropholis venulosa $\left(0,67 \mathrm{~m}^{2} . \mathrm{ha}^{-1} ; 79\right.$ indivíduos); Inga brevialata $\left(0,41 \mathrm{~m}^{2} . \mathrm{ha}^{-1}\right.$; 72 indivíduos); Licania incana $\left(10,57 \mathrm{~m}^{2} . \mathrm{ha}^{-1} ; 62\right.$ indivíduos) e Saccoglottis amazonica $\left(0,54 \mathrm{~m}^{2} . \mathrm{ha}^{-1} ; 51\right.$ indivíduos). Essas espécies também apresentaram as maiores densidades na área amostrada. 
Eschweilera blanchetiana apresentou o maior valor de importância $(26,01)$, seguida por Pouteria heterosepala $(25,40)$, ambas com altas densidade e dominância (Tabela 1). Eschweilera amazonica ficou em terceiro lugar $(18,17)$ devido à alta densidade. Um grupo formado por seis espécies (Manilkara paraensis, Micropholis venulosa, Protium apiculatum, Inga brevialata, Licania incana e Saccoglottis amazonica) também apresentou alta dominância.

\section{Características ecológicas e econômicas}

Quanto aos grupos ecológicos, quase dois terços das espécies encontradas $(63,29 \%)$ são tolerantes à sombra, seguidos das espécies intolerantes à sombra e as indefinidas (Figura 2). As espécies de uso comercial foram a maioria, seguidas pelas não-comerciais, potenciais e indefinidas (Figura 3). Entre as espécies comerciais, 35 são tolerantes à sombra, enquanto outras 30 são intolerantes à sombra ou demandantes de luz (Tabela 1). Entre as dez espécies sem definição de grupo ecológico ou uso comercial (Tabela 1), seis delas foi devido aos espécimes não terem sido identificados em nível taxonômico infragenérico. Sobre as demais espécies, embora identificadas, não encontraram-se informações suficientes na literatura para classificá-las.

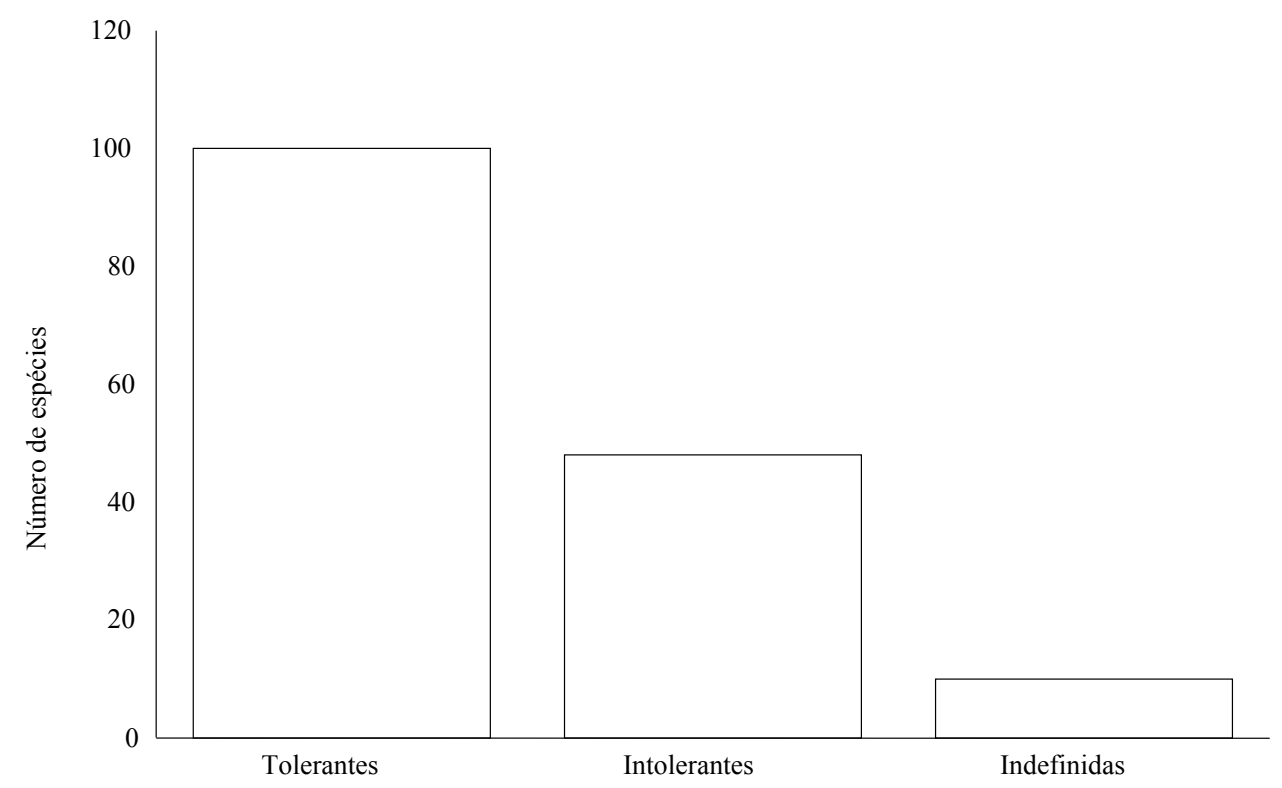

Figura 2. Grupos ecológicos das espécies inventariadas na fazenda Rio Capim. Espécies tolerantes à sombra, intolerantes à sombra e espécies indefinidas quanto ao grupo ecológico.

Figure 2. Ecological groups of inventoried species in Rio Capim farm. Shade-tolerants, shadeintolerants, and unknown species for ecological groups.

\section{DISCUSSÃO}

A família Fabaceae apresenta altas freqüências na região neotropical (WHITMORE, 1990; RIBEIRO et al., 1999a). Altas freqüências de Fabaceae foram encontradas por Barros et al. (2000) e Costa et al. (2002) no oeste do Pará. Para a Amazônia Central, além de Fabaceae, as famílias Lecythidaceae e Sapotaceae apresentam as maiores densidades de indivíduos e espécies (PRANCE, 1990; RANKIN-DE-MERONA et al., 1992; MILLIKEN, 1998; OLIVEIRA; AMARAL, 2004). O mesmo foi encontrado por Silva et al. (1992) e Andel (2001) para outras partes da Amazônia. No presente trabalho, as altas freqüências para essas famílias ficam confirmadas para o leste da Amazônia.

A raridade é um padrão comum para a maioria das comunidades na Floresta Amazônica (HUBBEL; FOSTER, 1986; HUSTON, 1995). Elevada raridade foi encontrada por Ribeiro et al. (1999b) no sul do Pará, cujos resultados apontaram $44 \%$ das famílias representadas por apenas uma espécie. As espécies raras, em determinadas regiões, podem apresentar características determinantes de suas baixas 
densidades. As famílias constituídas predominantemente por espécies pioneiras, as quais necessitam de muita luz (Araliaceae, Bixaceae, Combretaceae, Elaeocarpaceae, Malpighiaceae e Tiliaceae) e não conseguem desenvolver-se sob o dossel da floresta (SWAINE; WHITMORE, 1988), podem apresentar raridade. Em florestas primárias, indivíduos adultos dessas espécies seriam fruto de mudanças pretéritas no ambiente (DENSLOW; HARTSHORN, 1994; WALKER, 2000). Quanto à família Opiliaceae, geralmente ocorre em baixas densidades e com poucas espécies em florestas densas. Tal padrão foi verificado por Lopes (1993) em levantamentos de composição florística na Floresta Nacional do Tapajós (oeste do Pará). Algumas das famílias com baixas densidades apresentam muitas espécies de sub-bosque, mais freqüentes nos estratos intermediários (compreendidos nos intervalos de 5 a $20 \mathrm{~cm}$ de DAP) e raramente atingem grandes diâmetros, como Violaceae, por exemplo. Outras podem variar de acordo com a ocorrência geográfica, podendo apresentar alta densidade em alguns lugares e baixa em outros.

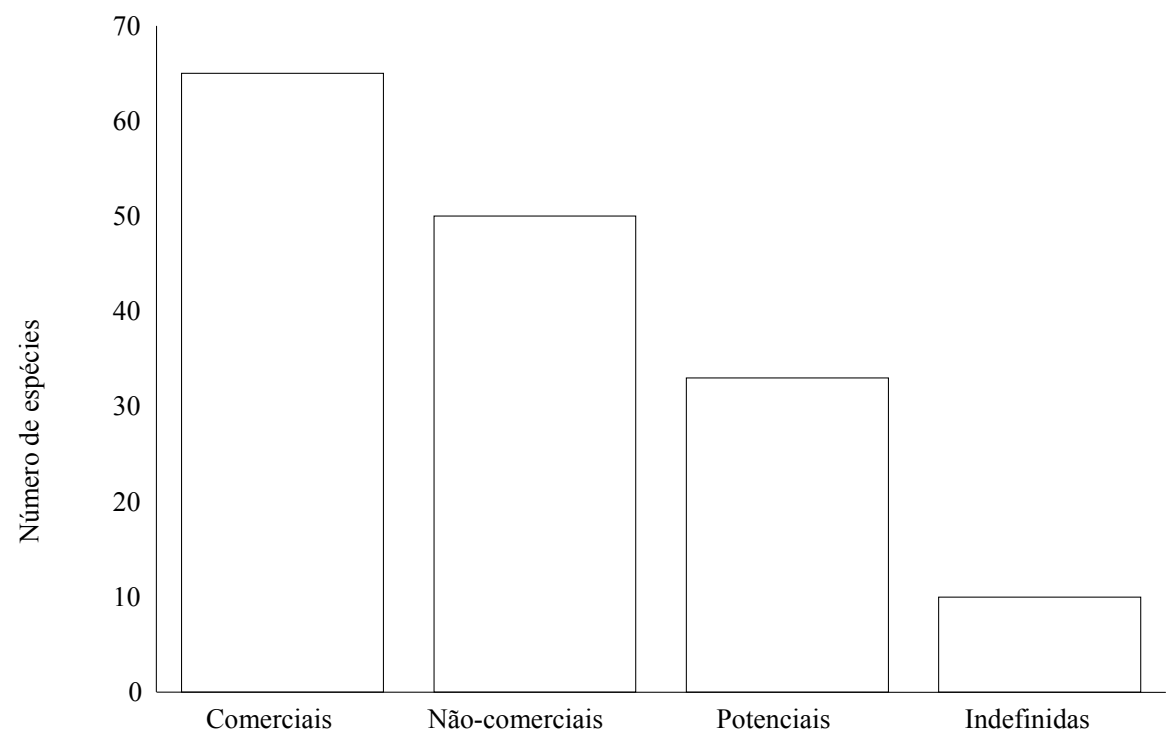

Figura 3. Uso comercial das espécies inventariadas na fazenda Rio Capim. Espécies comerciais, nãocomerciais, potenciais e espécies indefinidas quanto ao uso comercial.

Figure 3. Commercial use of inventoried species in Rio Capim farm. Commercials, non-commercials, potentials, and unknown species for commercial use.

\section{Implicações para o manejo}

Embora sem interesse comercial, as espécies Eschweilera amazonica, Eschweilera blanchetiana, Protium apiculatum, Guatteria poeppigiana, Sterculia pilosa, Inga brevialata e Licania heteromorpha devem ser consideradas em ações de conservação em ambientes semelhantes ao estudado. O alto valor de importância apresentado por elas pode estar indicando funções-chave que podem estar desempenhando nos ecossistemas que ocupam.

As espécies intolerantes à sombra, por terem rápido crescimento, podem ser utilizadas na recuperação de diferentes locais. Sua utilização é recomendável em áreas que, mesmo sendo consideradas de preservação permanente, já tenham sofrido algum tipo de alteração antrópica. Entre tais alterações, podem ocorrer as de descaracterização da vegetação ciliar em igarapés e córregos (KAGEYAMA; GANDARA, 2001). Esse tipo de alteração foi freqüente em áreas de pastagem na fazenda Rio Capim e adjacências (observação pessoal).

As espécies intolerantes à sombra que podem ser utilizadas para a recuperação de áreas alteradas em um primeiro estádio são: Inga brevialata, Inga paraensis, Piptadenia suaveolens, Sterculia pilosa, Tachigali myrmecophyla, Licania incana, Cordia alliodora e Anacardium giganteum. Em um segundo estádio, quando as espécies intolerantes à sombra estiverem estabelecidas e proporcionando sombreamento devido ao gradual fechamento do dossel, pode-se adensar a vegetação com as espécies 
tolerantes à sombra. Entre elas estão: Pouteria sp., Eschweilera amazonica, Eschweilera blanchetiana, Protium apiculatum, Guatteria poeppigiana, Duguetia echinophora, Rinorea flavescens, Eperua bijuga e Licania heteromorpha.

Bentes-Gama (2000), em área de várzea alta de topografia plana a suavemente ondulada destinada a projetos de manejo florestal, também encontrou as espécies Virola melinonii e Carapa guianensis no grupo das comerciais, e Symphonia globulifera, Tapirira guianensis e Saccoglottis guianensis no grupo das potenciais. No entanto, embora indicadores ecológicos apontem para a relevância de determinada espécie, alguns critérios devem ser pensados na escolha de espécies destinadas à recuperação de áreas alteradas ou degradadas. Para selecionar as espécies a serem utilizadas em programas de recuperação de áreas, entre elas as matas ciliares, devem-se realizar estudos do potencial biótico de cada espécie com base na produção e germinação de sementes, no estabelecimento das mudas, em pesquisas de viveiro e em silvicultura de plantações (MAGALHÃES; FERREIRA, 2000).

Entre as espécies com os melhores indicadores, ocorrem algumas com forte demanda comercial, tanto no mercado interno quanto externo. Nesse estudo destacam-se Manilkara paraensis, Piptadenia suaveolens, Carapa guianensis e Manilkara huberi. Por outro lado, algumas espécies têm grande potencial comercial, como Laetia procera e Tapirira guianensis (FINEGAN, 1992; ALVINO et al. 2005).

Neste estudo, algumas espécies de baixas densidades, como Manilkara paraensis, Carapa guianensis, Virola melinonii, Copaifera multijuga e Anacardium giganteum, apresentam importantes funções ecológicas e econômicas para as populações locais (MAGALHÃES; FERREIRA, 2000). Dessa forma, também se incluem entre as espécies sugeridas para a recuperação de áreas alteradas.

\section{REFERÊNCIAS}

ALVINO, F. O.; SILVA, M. F. F.; RAYOL, B. P. Potencial de uso das espécies arbóreas de uma floresta secundária, na Zona Bragantina, Pará, Brasil. Acta Amazonica, Manaus, v. 34, n. 4, p. 413-420, 2005.

ANDEL, T. Floristic composition and diversity of mixed primary and secondary forests in northwest Guyana. Biodiversity and Conservation, Amsterdam, v. 10, n. 10, p. 1645-1682, 2001.

APG II. An update of the Angiosperm Phylogeny Group classification for the orders and families of flowering plants: APG II. Botanical Journal of the Linnean Society, Londres, v. 141 n. 4, p. 399-436, 2003.

BARROS, A. V.; BARROS, P. L. C.; SILVA, L. C. B. Estudo da diversidade de espécies de uma floresta situada em Curuá-Una - Pará. Revista de Ciências Agrárias, Belém, v. 34, p. 49-65, 2000.

BENTES-GAMA, M. M. Estrutura, valoração e opção de manejo sustentado para uma floresta de várzea na Amazônia. 206 p. Dissertação (Mestrado em Engenharia Florestal) - Universidade Federal de Lavras, 2000.

BRASIL. Superintendência de Desenvolvimento da Amazônia. Grupamento de espécies tropicais da Amazônia por similaridade de características básicas e por utilização. Belém, 1981. 197 p.

CARVALHO, J. O. P. DE. 1992. Structure and dynamics of a logged over Brazilian Amazonian rain forest. $215 \mathrm{f}$. Tese (Doutorado) - Universidade de Oxford. 1992.

COSTA, D. H. M.; CARVALHO, J. O. P.; SILVA, J. N. M. Dinâmica da composição florística após a colheita de madeira em uma área de terra firme na Floresta Nacional do Tapajós (PA). Revista de Ciências Agrarias, Belém, v. 38, p. 67-80, 2002.

DENSLOW, J. S.; HARTSHORN, G. S. Tree-fall gap environments and forest dynamic processes. In: MCDADE, L. A.; BAWA, K. S.; HESPENHEIDE, H. A.; HARTSHORN, G. S. La Selva: ecology and natural history of a neotropical rain forest. Chicago: University of Chicago Press, 1994. p. 120-127.

FINEGAN, B. The management potential of neotropical secondary lowland rain forest. Forest Ecology and Management, Amsterdam, v. 47, p. 295-321, 1992. 
FÖRSTER, M. Strukturanalyse eines tropischen Regenwaldes in Kolumbien. Allgemeine Forst- und Jagdzeitung, Frankfurt, v. 144, n. 1, p.1-8, 1973.

HUBBELL, S. P.; FOSTER, R. B. Commonness and rarity in a neotropical forest: implications for tropical tree conservation In: SOULÉ M.E. (Ed.). Conservation Biology: the science of scarcity and diversity. Massachusetts: Sinauer. 1986. p. 205-231.

HUSTON, M. A. Biological diversity: the coexistence of species on changing landscapes. New York: Cambridge University Press. 1994. 681 p.

Instituto Brasileiro de Desenvolvimento Florestal. Madeiras da Amazônia: características e utilização. Brasília, DF, 1988. v. 2.

KAGEYAMA, P.; GANDARA, F. B. Recuperação de áreas ciliares. In: RODRIGUES, R. R.; LEITÃOFILHO, H. F. Matas ciliares: conservação e recuperação. São Paulo: EDUSP/Fapesp, 2001. p. 249-269.

KAO, D.; IIDA, S. Structural characteristics of logged evergreen forests in Preah Vihear, Cambodia, 3 years after logging. Forest Ecology and Management, Amsterdam v. 225, p. 62-73, 2006.

LOPES, J. C. A. Demografia e flutuação temporais da regeneração natural após uma exploração florestal: Flona do Tapajós - PA. 133 p. Dissertação (Mestrado) - Escola Superior de Agricultura Luiz de Queiroz, Universidade de São Paulo, Piracicaba, 1993.

MAGAlHÃES, C. S.; FERREIRA, R. M. A. Área de preservação permanente em uma microbacia. Informe Agropecuário, Belo Horizonte, v. 2, n. 207, p. 33-39, 2000.

MEDAUAR, O (Org.). Coletânea de legislação de Direito Ambiental: Constituição Federal. São Paulo: Revista dos Tribunais. 2002. 766 p.

MILARÉ, Direito do Ambiente: doutrina-prática-jurisprudência-glossários. São Paulo: Revista dos Tribunais. 2000. 76 p.

MILLIKEN, W. Structure and composition of one hectare of Central Amazon terra firme Forest. Biotropica, Malden, v. 30, n. 4, p. 27-38, 1998.

MORAIS-CRUIA, A. P. O., VEIGA, J. B.; LUDONINO, R. M. R.; SIMÃO-NETO, M.; TOURRAND, J. F. Caracterização dos sistemas de produção da agricultura familiar de Paragominas-PA: a pecuária e propostas de desenvolvimento. Belém: Embrapa Amazônia Oriental, 1999. 55 p. (Documentos, n. 5)

OLIVEIRA, A. N.; AMARAL, I. L. Florística e fitossociologia de uma floresta de vertente na Amazônia Central, Amazonas, Brasil. Acta Amazonica, Manaus, v. 34, n. 1, p. 21-34, 2004.

PRANCE, G. The floristic composition of the forest of Central Amazonian Brazil. In: Gentry, A. H. Four Neotropical Rain Forest. New Haven: Yale University Press, 1990. 146 p.

RANKIN-DE-MERONA, J.; PRANCE, G. T.; HUTCHINGS, R. W.; SILVA, M. F.; RODRÍGUEZ, W. A.; VENLING, M. E. Preliminary results of a large-scale tree inventory of upland rain forest in the Central Amazon. Acta Amazonica, Manaus, v. 22, n. 4, p. 485-492, 1992.

RIBEIRO, J. E. L. S.; HOPKINS, M. J. G.; VICENTINI, A.; SOTHERS, C. A.; COSTA, M. A. S.; BRITO, J. M.; SOUZA, M. A. D.; MARTINS, L. H. P.; LOHMANN, L. G.; ASSUNÇÃO, P. A. C. L.; PEREIRA, E. C.; SILVA, C. F.; MESQUITA, M. R.; PROCÓPIO, L. C. Flora da Reserva Ducke: guia de identificação das plantas vasculares de uma floresta de terra-firme na Amazônia Central. Manaus: Instituto Nacional de Pesquisas da Amazônia. 1999a. 799 p.

RIBEIRO, R. J.; HIGUCHI, J. S., AZEVEDO, C. P. Estudo fitossociológico nas regiões de Carajás e Marabá-Pará, Brasil. Acta Amazonica, Manaus, v. 29, n. 2, p. 207-222, 1999b.

RODRIGUES, R. R.; NAVE, A. G. Heterogeneidade florística das matas ciliares. In: RODRIGUES, R. R.; LEITÃO-FILHO, H. F. Matas ciliares: conservação e recuperação. São Paulo: EDUSP/Fapesp, 2001. p. 45-71. 
SANDEL, M. P.; CARVALHO, J. O. P. Composição florística e estrutura de uma área de cinco hectares de mata alta sem babaçu na Floresta Nacional do Tapajós. Belém: Embrapa Amazônia Oriental. 2000. 19 p. (Documentos, n. 63)

SANTANA, J. A. S.; ALMEIDA, W. C.; SOUSA, L. K. V. S. Florística e fitossociologia em área de vegetação secundária na Amazônia Oriental. Revista de Ciências Agrárias, Belém, v. 41, p. 105-120, 2004.

SHEPHERD, G. J. FITOPAC1: Manual do usuário. Campinas: Universidade Estadual de Campinas. 1994. $78 \mathrm{p}$.

SILVA, A. S. L.; LISBOA, P. L. B.; MACIEL, U. N. Diversidade florística e estrutura em floresta densa da bacia do Rio Juruá. Boletim do Museu Paraense Emílio Goeldi. Série Botânica, Belém, v. 8, n. 2, p. 203-258, 1992.

SILVA, A. S. L.; LOPES, J. C. A. Inventário florestal contínuo em florestas tropicais: a metodologia utilizada pela EMBRAPA-CPATU, na Amazônia brasileira. Belém: Embrapa Amazônia Oriental. 1984. (Documentos, n. 33)

SILVA, J. N. M.; LOPES, J. C. A.; OLIVEIRA, L. C.; SILVA, S. M. A.; CARVALHO, J. O. P.; COSTA, D. H. M.; MELO, M. S.; TAVARES, M. J. M. Diretrizes para instalação e medição de parcelas permanentes em florestas naturais da Amazônia Brasileira. Belém: Embrapa Amazônia Oriental. 2005. $68 \mathrm{p}$.

SILVA, M. F. F.; ROSA, N. A. Análise do estrato arbóreo da vegetação sobre jazidas de cobre na serra dos Carajás-PA. Boletim do Museu Paraense Emílio Goeldi. Série Botânica, Belém, v. 5, n. 2, p. 175$207,1989$.

SILVA, R. C. Contribuição do levantamento de solo a caracterização dos sistemas naturais e ambientais na região de Paragominas - Estado do Pará. 107 p. Dissertação (Mestrado) - Faculdade de Ciências Agrárias do Pará, Belém, 1997.

SOUZA, M. H.; MAGLIANO, M. M.; CAMARGOS, J. A. A.; SOUZA, M. R. Madeiras Tropicais Brasileiras - Brazilian tropical woods. Brasília, DF: IBAMA, 1997. 198 p.

SOUZA, P. F. Terminologia florestal - glossário de termos e expressões florestais. Rio de Janeiro: Fundação IBGE, 1973. 304 p.

SUDAM-DRN/EMBRAPA-SNLCS. Estudos climáticos do estado do Pará, classificação (Köppen) e deficiência hídrica (Thorntwaite, Mather). Belém: Superintendência de Desenvolvimento da Amazônia, 1993. 53 p.

SWAINE, M. D.; WHITMORE, T. C. On the definition of ecological species groups in tropical rain forests. Vegetatio, Amsterdam, v. 75, p. 81-86, 1988.

VELOSO, H. P.; RANGEL-FILHO, A. L. R.; LIMA, J. C. A. Classificação da vegetação brasileira, adaptada a um sistema universal. Rio de Janeiro: IBGE, 1991. $124 \mathrm{p}$.

WALKER, L. R. Seedling and sapling dynamics of treefall pits in Puerto Rico. Biotropica, Malden, v. 32, n. 2, p. 262-275, 2000.

WATRIN, O. S.; ROCHA, A. M. A. Levantamento da vegetação natural e do uso da terra no município de Paragominas (PA) utilizando imagens TM/ LANDSAT. Belém: Embrapa Amazônia Oriental, 1991. 40 p. (Boletim de Pesquisa, n. 124)

WHITMORE, T. C. Canopy gaps and the two major groups of forest trees. Ecology, Stanford, v. 70, n. 3 , p. 536-538, 1989a.

WHITMORE, T. C. Changes over twenty-one years in the Kolombangara rain forests. Journal of Ecology, London, v. 77, p. 469-483, 1989b. 
WHITMORE, T. C. An introduction to tropical rain forests. New York: Oxford. University Press. 1990. $198 \mathrm{p}$.

YARED, J. A. G.; CARVALHO, J. O. P.; SILVA, J. N. M.; KANASHIRO, M.; MARQUES, L. C. T. 2000. Contribuições do Projeto Silvicultura Tropical: Cooperação Internacional Brasil/Reino Unido. Belém: Embrapa Amazônia Oriental, 2000. 28 p. (Documentos, n. 52) 